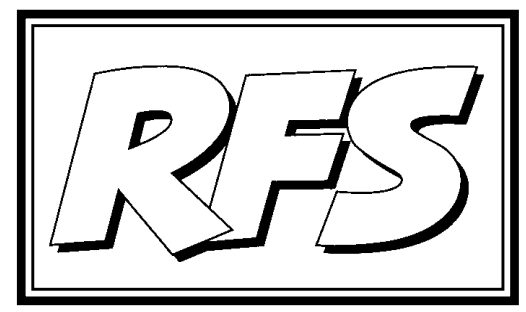

Revista de Fomento Social, 60 (2005), 57-86

\title{
Situación laboral y distribución del tiempo: un estudio empírico
}

José Antonio ARIZA, Mariano CARBONERO, Belén GUTIÉRREZ y M ${ }^{a}$ Carmen LÓPEZ ${ }^{1}$

(PALABRAS CLAVE: ACTIVO, INACTIVO, OCUPADO, DESEMPLEADO, TRABAJO PROFESIONAL, TRABAJO DOMÉSTICO, TIEMPO LIBRE)

(KEY WORDS: WORKERS, NON-WORKERS, EMPLOYED, UNEMPLOYED, PROFESSIONAL WORK, HOUSEWORK, FREE TIME)

\section{El tiempo: el recurso más valioso}

El tiempo es, probablemente, el bien más preciado que posee cualquier persona a lo largo de su vida. Aún más en las sociedades occidentales, donde el tiempo es como el dinero, se gasta, se invierte o incluso se despilfarra. Son varios los motivos que justifican tan tajante afirmación. En primer lugar, el tiempo es muy escaso. La esperanza de vida de un español varón es por tér-

1 Facultad de CC.EE. y Empresariales - ETEA (Universidad de Córdoba). 
mino medio de 74,4 años, lo que supone un total de 641.520 horas de vida. Si tiene la fortuna de ser mujer, entonces dispondrá de 63.360 horas más, ya que la esperanza de vida de una española es de 81,6 años. Por otra parte, la vida profesional ronda en la actualidad los 40 años. Si se plantea una jornada laboral de ocho horas al día y un total de 220 días laborables al año (1.760 horas), nos encontramos con 70.400 horas disponibles durante la vida para las actividades de naturaleza profesional. A determinadas personas este tiempo les parecerá mucho, pero, a la mayoría de la gente les resultará escaso para realizar todo aquello que han planificado. En segundo lugar, el tiempo es el capital más valioso, porque es un recurso precioso e irremplazable, no se puede comprar, ni adquirir ni, por supuesto, almacenar. En este sentido, el tiempo es un bien que no entiende de clases sociales. Se podría hablar de él como "el gran igualador". Todas las personas disponen de la misma cantidad de este recurso al día. El instante no vivido de la forma deseada no se puede recuperar de ningún modo. El tiempo pasa de forma lenta, pero inexorable. Además, presenta un elemento de incertidumbre elevado, es un bien que se consume sin conocer con exactitud cuando se va a agotar. En definitiva, el valor del tiempo radica en su propia esencia, ya que el tiempo es VIDA, nuestra vida.

Pese a que el valor del recurso tiempo es incuestionable, y que todas las personas comienzan el día con la misma cantidad, lo cierto es que el modo de consumirlo difiere de unos sujetos a otros. El tiempo, al igual que cualquier otro bien, no significa lo mismo para todo el mundo (Ariza, 2002). Las personas viven y disfrutan de su tiempo de una forma u otra en función del valor que conceden a las cosas. Sólo de esta manera se explica que algunos aficionados al fútbol hagan cola durante más de una semana en las taquillas del estadio de día y de noche, bajo el sol o la lluvia, con frío o calor, para obtener una entrada para un "partido del siglo" más entre los dos máximos aspirantes al campeonato. Otras personas, que a lo mejor descalifican a los anteriores, son capaces de volar durante más de ocho horas para asistir a una exposición que se celebra al otro lado del Océano Atlántico. Hay sujetos que se quejan de la escasez de tiempo, pero dedican muchas horas a actividades que para otros serían pueriles. Todo ello corrobora que la forma de perder/emplear el tiempo es distinta según los gustos y preferencias personales.

En conclusión, el valor que se concede al tiempo está condicionado por diferentes factores. Las investigaciones realizadas sobre el uso del tiempo han puesto de manifiesto cuáles son los elementos principales que determinan el modo de distribuir este bien. En el siguiente apartado haremos un breve recorrido sobre estos estudios. 


\section{Investigaciones empíricas sobre el empleo del tiempo}

La manera en que se emplea el tiempo adquiere una importancia y trascendencia incuestionable, de ahí que últimamente hayan proliferado los estudios que adoptan como objeto de análisis el modo de distribuir el tiempo de la población. La mayoría de estas investigaciones centran su atención en la población en su conjunto, distinguiendo, a lo sumo, por criterios de edad y sexo.

No obstante, las encuestas sobre el uso del tiempo de la población comenzaron mucho antes, concretamente a principios del siglo XX, desarrolladas en zonas muy localizadas como barrios y ciudades de Europa y Estados Unidos. Estos informes centran su atención en temas específicos vinculados a ciertas preocupaciones sociales como los medios económicos de vida en Inglaterra (Pember, 1913) o los modos de vida urbanos en EE.UU. (Lundberg, Komarovsky y McInery, 1934). Recientemente, Raldúa (2001), en su trabajo titulado Comparación internacional de los empleos del tiempo de mujeres y hombres, realiza un recorrido histórico sobre los estudios previos que existen en relación a este asunto, concediendo a Georges Esdras Bevas (Pronovost, 1989), Walker y Woods (Robinson, 1987) y al profesor Strumilin, el título de primeros investigadores del uso del tiempo. La generalización de este tipo de estudios se produjo durante la década de los años sesenta, vinculada al interés por conocer el empleo del tiempo de la audiencia de televisión. En la segunda mitad de esta década se desarrollan en España las primeras investigaciones, encargadas por RTVE (1965, 1968, 1972, 1973, 1976 y 1987) con la misma finalidad. En 1992, Telemadrid contrató una encuesta similar. Otros estudios, no vinculados directamente con la audiencia televisiva fueron los del Área Metropolitana de Barcelona (1987), el Instituto de la Juventud (1983 y 1988), el Instituto Vasco de Estadística (1993), el Instituto Nacional de Estadística (INE) (1996-1997), los del Centro de Investigaciones sobre la Realidad Social (CIRES) (1991 y 1996) y los del Centro Superior de Investigaciones Científicas (CSIC) (1990, 1994 y 1995).

Las grandes líneas de investigación sobre el uso del tiempo han adoptado, principalmente, una triple perspectiva: diacrónica, sincrónica y mixta. Las comparaciones diacrónicas analizan los cambios ocurridos en el modo de emplear el tiempo entre dos períodos diferentes ${ }^{2}$. Por su parte, las investi-

2 En esta categoría se pueden incluir los siguientes estudios: cambios en el uso del tiempo en Letonia entre 1972 y 1989 (ARTIOMOv, 1991; EgLITE y ZARINS, 1993); en Hungría entre 1963 y 1976-77 (ANDORKa y Falussy, 1982); en Estados Unidos entre 1965 y 1975 (RoBinson, 1985); en 
gaciones sincrónicas pretenden establecer comparaciones entre diferentes países con relación al modo de utilizar el tiempo disponible. En este sentido resulta de mención obligada el informe The Use of Time, dirigido por Alexander Szalai y enmarcado dentro un gran proyecto internacional donde participaron doce países. El objetivo de este estudio era armonizar a nivel supranacional las investigaciones sobre el empleo y distribución del tiempo de la población. Este informe es una referencia obligada para cualquier estudioso del tema (Szalai y otros, 1972). Por último, Jonathan Gershuny abandera a mediados de los años ochenta una nueva línea de investigación que integra las dos perspectivas anteriores. Sus publicaciones explotan los datos del Multinational Longitudinal Time Budget Archive (MLTBA), un archivo sobre el uso del tiempo patrocinado por la Fundación Europea para la Mejora de las Condiciones de Vida y Trabajo ${ }^{3}$.

En otro orden de cosas, hay que advertir que la mayoría de los estudios realizados hasta el momento han centrado su atención, sobre todo, en las diferencias existentes entre hombres y mujeres en relación con el uso del tiempo. En casi todos los casos las conclusiones son coincidentes y obvias: los hombres emplean más tiempo en el trabajo remunerado, mientras que las mujeres se dedican con mayor énfasis a tareas no remuneradas. Pero, en general, el trabajo total de éstas -incluyendo cualquier trabajo con independencia de su carácter remunerado o no- es superior al de los hombres. Tal circunstancia origina que la población masculina disfrute, generalmente, de más tiempo libre ${ }^{4}$.

Australia entre 1974 y 1987 (BITTMAn, 1993); en Japón entre 1960 y 1975 (ANDORKA, 1987); en Noruega entre 1971-72 y 1975 (SAMUEL, 1986) y entre 1975 y 1985 (Roy, 1991); y en España entre 1976 y 1987 (RALDÚA, 1997).

3 Algunas de las obras resultantes son las siguientes: GERSHUNy y Jones (1986), GeRSHUNy (1987), GERSHUNy (1991), GeRSHUNy (1992) y GeRShUNy (1993).

4 Esta afirmación se contrasta en varios de los estudios más importantes elaborados a nivel nacional. Por ejemplo, utilizando una muestra de personas de 14 años y más, RAmos (1990) concluye que en un día promedio de la semana el hombre dispone de casi dos horas más al día que las mujeres para disfrutar del tiempo libre (7,23 horas frente a 5,33). En el mismo sentido, PAGE (1996) apunta -con una muestra integrada por personas de 18 años y más- que un varón dedica por término medio 9,27 horas/día al tiempo libre, frente a las 8,61 horas/día de las que dispone una mujer. Pese a utilizar exclusivamente una muestra de personas -hombres o mujeres- que realizan trabajo remunerado, el estudio del Instituto Vasco de Estadística-EUSTAT (1998) llega a conclusiones similares: sumando todo el tiempo dedicado a las diferentes actividades productivas, remuneradas o no (trabajo, formación, tareas domésticas, cuidados a personas y trayectos), nos encontramos con que las mujeres trabajan 
Como se puede comprobar, el sexo es una variable de incidencia indiscutible en el modo de distribuir el tiempo, pero no es la única. Szalai (1972) identifica, además de ésta, al estatus familiar y la presencia o no de hijos, por un lado; y por otro, la situación laboral, el estatus profesional y el tipo de día (laborable o festivo). Harvey y otros (1977) consideran como principales variables de influencia el sexo, el día de la semana, el ciclo vital del individuo, la edad, la educación, la relación con el cabeza de familia y el tipo de día. Aas (1982) señala como variables críticas el sexo, la situación laboral y el rol familiar. Por su parte, Clark, Elliot y Harvey (1982) apuntan al sexo, la situación laboral, el estado civil y la presencia de hijos en el seno familiar como los elementos de mayor importancia en la forma de distribución del tiempo. Izquierdo, del Río y Rodríguez (1988) afirman que el sexo es la variable más determinante, ya que condiciona la realización del trabajo doméstico.

Tal y como se ha podido apreciar, la mayoría de las investigaciones previas han volcado su interés en aspectos como el sexo, la situación familiar, o el estado civil, entre otros. Sin embargo, una de las variables de mayor alcance a priori -la situación laboral del individuo- no ha sido objeto de un estudio profundo y detallado. Precisamente, nuestra investigación adopta como eje principal de análisis esta cuestión, es decir, el análisis de las diferencias en el uso del tiempo en función de la situación laboral en que se encuentre el individuo. Para ello, como se expondrá a continuación en la metodología, se utilizarán las propuestas de la Encuesta de Población Activa (EPA), que sigue las recomendaciones efectuadas a nivel mundial por la Organización Internacional del Trabajo ${ }^{5}$.

diariamente 59 minutos más que los hombres. La contrapartida no puede ser otra, las mujeres disponen de 59 minutos diarios menos que los hombres para dedicarlos a las actividades de ocio y de relación (p. 3). A nivel internacional, la comparación sincrónica efectuada por RALDÚa (2001) con datos provenientes de la División de Estadísticas de las Naciones Unidas de 1997, reveló que las mayores diferencias se producían en España (12,1 horas/semana), Italia (9,3 horas/semana), Bulgaria (9 horas/semana) y Polonia (7,9 horas/semana).

5 El Instituto Nacional deEstadística (INE) ha elaborado una investigación que adopta, en parte, un enfoque similar al aquí propuesto. Se trata de una encuesta dirigida a una muestra de unos 24.000 hogares, que recaba información sobre las actividades diarias de las personas a través de la cumplimentación de diarios personales y cuestionarios de hogar e individuales. El procedimiento empleado consiste en rellenar el diario de actividades por todos los miembros del hogar seleccionados de 10 y más años en un día concreto. 


\section{Metodología}

\subsection{Diseño de la muestra y recogida de la información}

El presente trabajo forman parte de una investigación más amplia cuyo objetivo fue el de analizar la realidad sociolaboral del municipio de Córdoba, empleando una metodología similar a la que se usa en la Encuesta de Población Activa (EPA). Los resultados se han elaborado a partir de la información obtenida de 1.400 entrevistas realizadas a domicilio entre los habitantes de la ciudad de Córdoba con 16 o más años. El trabajo de campo se realizó durante el primer trimestre de 2003.

Con objeto de garantizar en lo posible la generalidad de las conclusiones, y ante la evidente imposibilidad de disponer de un listado con la identificación de todos los miembros del universo investigado, la muestra fue obtenida mediante el sistema de cuotas de edad y sexo, posiblemente las dos variables sociodemográficas que mejor definen la estructura de una población. Por otra parte, y para evitar el posible efecto socioeconómico que pudiera derivarse de la concentración de entrevistas en determinadas zonas del municipio, las visitas se distribuyeron entre los ocho distritos en que la ciudad se divide, de manera proporcional a su población. Finalmente, tras la depuración de resultados, fue necesario prescindir de cien de los cuestionarios obtenidos, por ser inconsistentes sus respuestas. De esta forma, se trabajó con un total de 1.300 observaciones válidas.

\subsection{Variables}

Para cada uno de los entrevistados se obtuvo la siguiente información:

a) Sexo y edad (esta última variable se agrupó en tres categorías: de 16 a 29 años, de 30 a 44 años y personas con 45 años o más).

b) Estado civil, según la siguiente clasificación: solteros, viudos, separados/divorciados y personas que conviven en pareja (matrimonio civil, religioso o parejas de hecho).

c) Nivel de formación: personas sin estudios o con estudios primarios, con formación secundaria (bachillerato, formación profesional o similar) y, finalmente, titulados superiores de cualquier grado. 
d) Ingresos mensuales. Dada la sensibilidad de la cuestión, se optó por solicitar del entrevistado que se encuadrara en alguno de los grupos que se le presentaban, lo suficientemente amplios como para eliminar la mayor parte de las reticencias. Concretamente, las categorías consideradas en función de la realidad cordobesa han sido las siguientes: menos de $781,32 €$; de 781,32 a $1.081,82 €$; de $1.081,82$ a $1.382,33 €$ y más de $1.382,33 €$.

e) Relación de la actividad. En este apartado se determina la situación del entrevistado, encuadrándolo en alguna de las siguientes categorías: ocupado, parado, estudiante, persona dedicada a las labores del hogar, jubilado/retirado o inactivo por otras circunstancias.

f) Distribución del tiempo. Se pidió a cada uno de los encuestados que indicara cuál era, en promedio, el tiempo que dedicaba semanalmente a cada una de las siguientes actividades: trabajo profesional, trabajo doméstico, formación, satisfacción de las necesidades básicas y tiempo libre. Si bien los resultados se presentan en términos diarios, resultaba imprescindible que el cómputo fuera semanal, para incluir las evidentes diferencias entre, al menos, días laborales y festivos.

\subsection{Metodología}

En términos estadísticos, la comparación de los valores de una variable en dos o más grupos de individuos, a partir de la evidencia obtenida de una parte de los mismos, puede llevarse a cabo desde dos puntos de vista distintos.

El primero, que podemos llamar descriptivo, se fundamenta en la observación de los principales indicadores estadísticos (normalmente medias y a lo sumo desviaciones típicas, sin establecer hipótesis sobre el fenómeno que va a ser objeto de análisis). Las diferencias observadas en estos indicadores entre los distintos grupos bajo estudio pueden ser empleadas para llegar a conclusiones, más o menos generales, sobre el fenómeno observado. Aunque muy sencillo, este procedimiento presenta como mínimo dos inconvenientes insalvables: por una parte, su alto grado de subjetivismo, que deja en manos del intérprete la decisión sobre la relevancia o no de las diferencias que necesariamente se encontrarán en todos los casos (resultado de la práctica imposibilidad de que las medias observadas para dos grupos cualesquiera de individuos analizados sean idénticas); por otra, la 
dificultad añadida que supone llevar a cabo, mediante este procedimiento, comparaciones entre más de dos grupos, dado el hecho de que éstas, por su propia naturaleza, habrán de extenderse necesariamente más allá de las simples comparaciones por pares.

El segundo punto de vista, que podemos llamar inferencial, es más complejo desde el ámbito teórico, sin embargo, resuelve los inconvenientes anteriores, estableciendo determinadas hipótesis sobre el fenómeno observado. Desde la perspectiva inferencial, las consecuencias derivadas del estudio de los datos procedentes de los individuos observados (muestras), pueden ser proyectadas (inferidas) al conjunto de las poblaciones de las que han sido extraídas, lo que permitirá que las conclusiones obtenidas vayan más allá de los mismos. No obstante, y dado que difícilmente la observación de una parte puede llevar a conclusiones ciertas sobre el todo, cualquier afirmación efectuada mediante este procedimiento correrá el peligro de ser errónea con mayor o menor probabilidad, siendo el conocimiento exacto de estas probabilidades (o al menos de límites precisos para las mismas), la mayor aportación del enfoque inferencial frente al descriptivo, al eliminar el subjetivismo en las conclusiones.

Uno de los procedimientos inferenciales más usual es el contraste de hipótesis, cuyo objetivo es la verificación de una determinada afirmación efectuada sobre la población o poblaciones analizadas, a partir de la evidencia aportada por las correspondientes muestras. Esta herramienta es la más apropiada para el estudio que nos ocupa, donde se pretende evaluar las diferencias existentes en el uso del tiempo en función de diversas características físicas (sexo, edad...) o sociales (estado civil, actividad económica...).

El tipo de contraste estadístico que emplearemos a continuación está condicionado por la cantidad de grupos a comparar, tal y como se detalla a continuación:

a) Comparación entre dos grupos. En esta situación se utilizan los estadísticos paramétricos $\mathrm{t}$ de Student, cuya formulación depende de que puedan o no considerarse iguales las varianzas en ambos grupos (prueba de Levene). Cuando no resulta aceptable la hipótesis sobre la igualdad de varianzas, la diferencia entre valores descriptivos puede ser interpretada en términos poblacionales.

b) Comparaciones entre tres o más grupos. En este caso se han empleado técnicas de Análisis de la Varianza (ANOVA), aunque éstas son diferentes 
en función de que resulte o no aceptable la hipótesis sobre la igualdad de varianzas en los distintos grupos, contrastada con el mismo estadístico indicado en el punto anterior. Cuando este supuesto resulta aceptable, se emplea el estadístico paramétrico F, sustituido por las pruebas no paramétricas de Brown-Forshythe y de Welch cuando no lo ha sido. Para analizar las diferencias entre los grupos, cuando se ha diagnosticado su existencia, se usan las pruebas de comparaciones múltiples de Student-Newman-Keuls y T3 de Dunnett, según se emplee o no la prueba $\mathrm{F}$ respectivamente.

Finalmente, y en todos los casos, se ha partido del supuesto de normalidad en las distintas distribuciones consideradas, usual en la aplicación de estos procedimientos.

\section{Resultados}

\subsection{Introducción}

Dado que el eje principal de nuestra investigación es la relación entre el empleo del tiempo y la situación laboral, resulta necesario definir cuáles son las realidades posibles en este sentido. De acuerdo con los criterios y recomendaciones estipuladas por la Organización Internacional del Trabajo, este análisis se realizará en tres niveles distintos. En primer lugar, se establece una comparación entre las características que definen a la población activa frente a la población inactiva. A continuación, centrando la atención en los activos, se evaluarán las diferencias existentes entre ocupados y desempleados. Finalmente, volcando todo el interés en la población inactiva, se mostrarán las disimilitudes que muestran los tres grupos principales de inactivos: estudiantes, pensionistas y personas dedicadas a las labores del hogar. Aunque algunas de las conclusiones obtenidas pueden parecer obvias, el valor del presente estudio radica en la cuantificación y validación estadística de las mismas.

Pero antes de profundizar en ello, a continuación caracterizaremos la población objeto de estudio de manera genérica, atendiendo a los criterios siguientes: sexo, edad, estado civil, formación y nivel de ingresos. 


\subsection{Distribución general}

El tiempo a lo largo de un día promedio en la muestra analizada se distribuye tal y como se puede apreciar en la zona sombreada del Cuadro 1. Cada persona dedica 1,92 horas al trabajo profesional, 3,92 horas a las labores domésticas, 0,97 horas a las actividades de formación, emplea 8,38 horas en satisfacer las necesidades básicas y, finalmente, disfruta de 8,79 horas al día de tiempo libre.

En comparación con los avances suministrados por la encuesta de empleo del tiempo del INE se aprecian notables diferencias. Por término medio, un español dedica más tiempo al trabajo (2:39 horas/día) y al cuidado personal (11:22 horas/día). Sin embargo, el tiempo de estudio es ligeramente inferior (0:49 horas/día), así como el tiempo destinado a las tareas domésticas (3:04 horas/día) y a las actividades de ocio (4:55 horas/día). Estas diferencias pueden estar sustentadas en dos motivos fundamentales. Por un lado, la investigación del INE integra a todas aquellas personas con 10 y más años, mientras que en el presente estudio se considera sólo a la población potencialmente activa (16 años o más). Por otro, es posible que exista cierta confusión a la hora de clasificar determinadas actividades como necesidades básicas o tiempo libre. Esta circunstancia ha podido provocar que, aunque individualmente resulten diferentes, en su conjunto ocupen la misma porción de tiempo, en torno a 16 horas promedio en cómputo diario.

Los resultados del estudio de Page (1996) con población de 18 y más años -por tanto, más parecida a la que se utiliza en este trabajo- se asimilan en mayor medida a la distribución que hemos obtenido: necesidades personales (10,35 horas/día), trabajo doméstico (5,34 horas/día), estudio ( 0,73 horas/día), trabajo remunerado (2,15 horas/día) y tiempo libre $(8,61 \text { horas/día })^{6}$.

6 En este estudio se permitía compatibilizar dos o más actividades al mismo tiempo. 
CUADRO 1. Distribución del tiempo promedio diario por sexo

\begin{tabular}{|c|c|c|c|c|}
\hline Variables & Sexo & Individuos & Media & Desviación típica \\
\hline \multirow{3}{*}{$\begin{array}{l}\text { Trabajo } \\
\text { profesional }\end{array}$} & Varones & 617 & 2,77 & 3,119 \\
\hline & Mujeres & 683 & 1,15 & 2,253 \\
\hline & Total & 1.300 & 1,92 & 2,816 \\
\hline \multirow{3}{*}{$\begin{array}{l}\text { Trabajo } \\
\text { doméstico }\end{array}$} & Varones & 617 & 2,28 & 2,098 \\
\hline & Mujeres & 683 & 5,41 & 2,435 \\
\hline & Total & 1.300 & 3,92 & 2,764 \\
\hline \multirow{3}{*}{ Formación } & Varones & 617 & 1,13 & 2,309 \\
\hline & Mujeres & 683 & 0,81 & 2,000 \\
\hline & Total & 1.300 & 0,97 & 2,157 \\
\hline \multirow{3}{*}{$\begin{array}{l}\text { Necesidades } \\
\text { básicas }\end{array}$} & Varones & 617 & 8,38 & 1,519 \\
\hline & Mujeres & 683 & 8,38 & 1,306 \\
\hline & Total & 1.300 & 8,38 & 1,410 \\
\hline \multirow{3}{*}{ Tiempo libre } & Varones & 617 & 9,41 & 2,990 \\
\hline & Mujeres & 683 & 8,22 & 2,311 \\
\hline & Total & 1300 & 8,79 & 2,720 \\
\hline
\end{tabular}

Fuente: elaboración propia.

\subsubsection{Sexo}

En el Cuadro 1 también se muestran las diferencias de género. Como era presumible, éstas son estadísticamente significativas en algunos aspectos, sobre todo en el tiempo dedicado a las tareas domésticas. En esta actividad, una mujer emplea tres horas más al día que un hombre (5,41 frente a 2,28 horas/día). La mayor disponibilidad de tiempo la dedica el varón al trabajo profesional (1,16 horas/día más que la mujer) y al tiempo libre (un hombre dispone de 1,19 horas al día más que una mujer para las actividades de ocio y diversión) ${ }^{7}$. En todos los casos, las diferencias existentes entre hombres y

$7 \quad$ En el estudio del INE, las diferencias de género también son notables, fundamentalmente en las variables trabajo profesional (3:37 horas diarias los hombres; 1:47 horas/día las mujeres), trabajo doméstico $(1: 29 ; 4: 29)$ y tiempo libre $(5: 28 ; 4: 31)$. Sin embargo, se aprecian comportamientos muy similares en el tiempo destinado a formación $(0: 49 ; 0: 48)$ y a la satisfacción 
mujeres -analizadas mediante la prueba t de Student-son estadísticamente significativas (Sig. 0,000), a excepción del tiempo dedicado a satisfacer las necesidades básicas (Sig. 0,987). En este caso, ambos géneros utilizan la misma cantidad del recurso tiempo: 8,38 horas al día.

\subsubsection{Edad}

Este análisis se efectuó segmentando la muestra en tres tramos de edad: de 16 a 29 años, de 30 a 44 años y personas con 45 años o más. Es conveniente recordar de nuevo que la muestra empleada sólo incluye a individuos que hayan cumplido los 16 años, es decir, que tengan la edad legal para acceder al mercado de trabajo.

Como se puede apreciar en el Cuadro 2, los jóvenes destacan por ser los que más tiempo destinan a la formación (2,85 horas/día), a la vez que constituyen el grupo con menor dedicación al trabajo profesional (1,55 horas/día) y doméstico (2,66 horas/día). Por su parte, las personas con edades comprendidas entre los 30 y 44 años se caracterizan, sobre todo, por su entrega al trabajo profesional (2,79 horas/día). Asimismo, presentan una gran dedicación a las tareas del hogar (4,22 horas/día) -tan sólo superados en esta faceta por los sujetos de edad más avanzada- y configuran el grupo con menos tiempo libre a su disposición (8,31 horas/día). Por último, el grupo de más edad sobresale por su dedicación al trabajo doméstico $(4,43$ horas/día), por disfrutar de más tiempo libre que nadie (9,18 horas/día), y también por disponer de algo más de tiempo para sus necesidades fundamentales $(8,48 \text { horas/día })^{8}$.

de las necesidades básicas (11:24; 11:20). El estudio de Page (1996) muestra una tendencia semejante, aunque aún es más escandalosa la distancia que separa a hombres y mujeres en cuanto a la dedicación a las tareas domésticas: 2,50 frente a 7,96 horas/día.

8 La variable edad en la investigación del INE se agrupó en torno a cuatro categorías: a) menores de 25 años, b) 25 a 44 años, c) 45 a 65 años, y d) 65 y más años. Los resultados obtenidos fueron los siguientes: trabajo profesional (1:28; 4:13; 3:07; y 0:07 horas/día, respectivamente); trabajo doméstico (1:02; 3:18; 3:43; y 3:37 horas/día); formación (3:48; 0:18; 0:04; 0:02 horas/día); necesidades básicas (11:31; 10:47; 11:10; y 12:38 horas/día); y tiempo libre (4:50; 4:00; 4:52; y 6:56 horas/día). 
CUADRO 2. Distribución del tiempo promedio diario por grupos de edad

\begin{tabular}{|c|c|c|c|c|}
\hline Variables & Grupos de edad & Individuos & Media & $\begin{array}{c}\text { Desviación } \\
\text { típica }\end{array}$ \\
\hline \multirow{4}{*}{$\begin{array}{l}\text { Trabajo } \\
\text { profesional }\end{array}$} & De 16 a 29 años & 330 & 1,55 & 2,648 \\
\hline & De 30 a 44 años & 365 & 2,79 & 3,044 \\
\hline & De 45 o más años & 605 & 1,60 & 2,647 \\
\hline & Total & 1.300 & 1,92 & 2,816 \\
\hline \multirow{4}{*}{$\begin{array}{l}\text { Trabajo } \\
\text { doméstico }\end{array}$} & De 16 a 29 años & 330 & 2,66 & 2,524 \\
\hline & De 30 a 44 años & 365 & 4,22 & 2,713 \\
\hline & De 45 o más años & 605 & 4,43 & 2,710 \\
\hline & Total & 1.300 & 3,92 & 2,764 \\
\hline \multirow{4}{*}{ Formación } & De 16 a 29 años & 330 & 2,85 & 3,134 \\
\hline & De 30 a 44 años & 365 & 0,40 & 1,272 \\
\hline & De 45 o más años & 605 & 0,28 & 1,041 \\
\hline & Total & 1.300 & 0,97 & 2,157 \\
\hline \multirow{4}{*}{$\begin{array}{l}\text { Necesidades } \\
\text { básicas }\end{array}$} & De 16 a 29 años & 330 & 8,34 & 1,422 \\
\hline & De 30 a 44 años & 365 & 8,26 & 1,418 \\
\hline & De 45 o más años & 605 & 8,48 & 1,394 \\
\hline & Total & 1.300 & 8,38 & 1,410 \\
\hline \multirow{4}{*}{ Tiempo libre } & De 16 a 29 años & 330 & 8,58 & 2,683 \\
\hline & De 30 a 44 años & 365 & 8,31 & 2,609 \\
\hline & De 45 o más años & 605 & 9,18 & 2,751 \\
\hline & Total & 1.300 & 8,79 & 2,720 \\
\hline
\end{tabular}

Fuente: elaboración propia.

Para el análisis estadístico posterior, necesario para valorar la significación de las diferencias detectadas, se ha hecho uso del Análisis de Varianzas (ANOVA). La contrastada homogeneidad de varianzas en las variables trabajo doméstico, necesidades básicas y tiempo libre nos ha llevado en estos casos al uso de técnicas de análisis paramétricos. En los dos restantes (trabajo profesional y formación), no resultó posible aceptar el supuesto de igualdad de las varianzas, lo que nos ha llevado a recurrir a estadísticos de contraste no paramétricos. 
De este modo, el análisis confirmó la existencia de diferencias entre los grupos para las tres variables analizadas: trabajo doméstico ( $\mathrm{F}=50,421$; Sig. 0,000), necesidades básicas ( $F=3,170$; Sig. 0,042) y tiempo libre $(F=13,161$; Sig. 0,000).

En el caso del trabajo doméstico, estas diferencias se producen entre el grupo de menor edad -que se dedica a esta actividad con menor intensidad (2,66 horas día) - y los dos restantes. Las personas de 30 a 44 años y los individuos de 45 años o más, distribuyen el tiempo dedicado a las tareas domésticas de forma muy similar: 4,22 y 4,43 horas/día respectivamente.

Por lo que respecta a las necesidades básicas, si bien la prueba ANOVA presenta evidencias de diferencias entre los distintos grupos de edad, el contraste de comparaciones múltiples posterior no logra detectar cuáles son éstas. Esta situación, que debe atribuirse a la relativamente elevada significación de la prueba correspondiente $(0,042)$, señala que si bien existen razones para suponer que existen diferencias, la evidencia disponible no es suficiente para concretar cuáles son.

Finalmente, en relación con el tiempo libre se ha confirmado que el comportamiento del grupo de mayor edad (9,18 horas al día disponibles) es diferente a los dos restantes, entre los que no existen diferencias: 8,58 horas/día en los más jóvenes y 8,31 horas/día en el grupo de 30 a 44 años.

Por otra parte, las variables trabajo profesional y formación se analizaron mediante las pruebas no paramétricas de igualdad de medias de Welch y Brown-Forsythe. Ambos estadísticos confirman la existencia de diferencias significativas según los grupos de edad. En relación al trabajo profesional, los individuos con edades comprendidas entre 30 y 44 años (2,79 horas al día empleadas en actividades profesionales) presentan diferencias significativas con los restantes grupos (Sig. 0,000 para ambos estadísticos). Aunque seguramente subyacen motivos diferentes, el tiempo dedicado a esta actividad entre los más jóvenes (1,55 horas/día) es similar al que aplican los mayores de 45 años (1,60 horas/día; Sig. 0,991).

Por lo que respecta a la formación, existen diferencias significativas entre los más jóvenes y el resto de grupos. Entre las personas de 16 a 29 años es más probable que abunde la población estudiantil, lo que origina que, por término medio, dediquen 2,85 horas al día a estos menesteres. Sin embargo, el tiempo dedicado a la formación entre las personas de 30 a 44 años y los de 45 años o más es semejante (Sig. 0,361) y sensiblemente inferior con respecto a los jóvenes ( 0,4 y 0,28 horas/día respectivamente). 


\subsubsection{Estado civil}

La clasificación de la muestra según su estado civil distingue entre las siguientes categorías: solteros, viudos, separados/divorciados y personas que conviven en pareja (matrimonio civil, religioso o parejas de hecho). Como se puede observar en el Cuadro 3, los separados se vuelcan con mayor profusión en la actividad profesional (2,43 horas/día por término medio), aunque también constituyen el grupo con mayor tiempo libre disponible (9,11 horas/día), seguidos de cerca por los solteros (8,85 horas/día). Estos últimos, seguramente por estar integrados mayoritariamente por jóvenes, destacan también por su dedicación a la formación (2,48 horas/día), a gran distancia del resto de grupos. Los viudos, por su parte, son los individuos que más tiempo aplican en tareas del hogar (5,64 horas/día). Finalmente, la distribución promedio del grupo más numeroso -o sea, el de las personas que viven en pareja- es la siguiente: trabajo profesional (2,09 horas/día), tareas domésticas ( 4,45 horas/día), formación ( 0,31 horas/día), necesidades básicas ( 8,38 horas/día) y tiempo libre $(8,76 \text { horas/día) })^{9}$.

CUADRO 3. Distribución del tiempo promedio diario según el estado civil

\begin{tabular}{|l|l|c|c|c|}
\hline Variables & \multicolumn{1}{|c|}{ Estado civil } & Individuos & Media & $\begin{array}{c}\text { Desviación } \\
\text { típica }\end{array}$ \\
\hline \multirow{4}{*}{$\begin{array}{l}\text { Trabajo } \\
\text { profesional }\end{array}$} & Solteros & 397 & 1,60 & 2,618 \\
\cline { 2 - 5 } & En pareja & 834 & 2,09 & 2,895 \\
\cline { 2 - 5 } & Viudos & 43 & 1,30 & 2,471 \\
\cline { 2 - 5 } & Separados/divorciados & 26 & 2,43 & 3,248 \\
\cline { 2 - 5 } & Total & 1.300 & 1,92 & 2,816 \\
\hline \multirow{4}{*}{$\begin{array}{l}\text { Trabajo } \\
\text { doméstico }\end{array}$} & Solteros & 397 & 2,60 & 2,303 \\
\cline { 2 - 5 } & En pareja & 834 & 4,45 & 2,743 \\
\cline { 2 - 5 } & Viudos & 43 & 5,64 & 1,808 \\
\cline { 2 - 5 } & Separados/divorciados & 26 & 4,52 & 3,753 \\
\cline { 2 - 5 } & Total & 1.300 & 3,92 & 2,764 \\
\hline
\end{tabular}

9 En relación con el estado civil, la investigación del Instituto Nacional de Estadística establece cuatro categorías: a) casado/a, b) soltero/a, c) viudo/a y d) separado/a y divorciado/a. Los 


\begin{tabular}{|c|c|c|c|c|}
\hline Variables & Estado civil & Individuos & Media & $\begin{array}{l}\text { Desviación } \\
\text { típica }\end{array}$ \\
\hline \multirow{5}{*}{ Formación } & Solteros & 397 & 2,47 & 3,048 \\
\hline & En pareja & 834 & 0,31 & 1,106 \\
\hline & Viudos & 43 & 0,21 & 0,642 \\
\hline & Separados/divorciados & 26 & 0,27 & 1,144 \\
\hline & Total & 1.300 & 0,97 & 2,157 \\
\hline \multirow{5}{*}{$\begin{array}{l}\text { Necesidades } \\
\text { básicas }\end{array}$} & Solteros & 397 & 8,45 & 1,382 \\
\hline & En pareja & 834 & 8,38 & 1,373 \\
\hline & Viudos & 43 & 8,23 & 1,825 \\
\hline & Separados/divorciados & 26 & 7,65 & 2,011 \\
\hline & Total & 1.300 & 8,38 & 1,410 \\
\hline \multirow{5}{*}{$\begin{array}{l}\text { Tiempo } \\
\text { libre }\end{array}$} & Solteros & 397 & 8,85 & 2,791 \\
\hline & En pareja & 834 & 8,75 & 2,712 \\
\hline & Viudos & 43 & 8,60 & 2,214 \\
\hline & Separados/divorciados & 26 & 9,11 & 2,725 \\
\hline & Total & 1.300 & 8,79 & 2,720 \\
\hline
\end{tabular}

Fuente: elaboración propia.

En este caso sólo se pudo recurrir al análisis paramétrico con la variable tiempo libre. Con el resto hubo de aplicarse pruebas de carácter no paramétrico.

En relación con la variable tiempo libre, el análisis ANOVA confirma que no existen diferencias significativas entre ninguno de los grupos analizados (Sig. 0,817), es decir, el estado civil no está relacionado de ningún modo con la distribución del tiempo de ocio.

resultados se muestran a continuación: trabajo profesional $(7: 46 ; 7: 39 ; 7: 21 ;$ y 7:25 horas/día, respectivamente); trabajo doméstico ( $4: 25 ; 2: 02 ; 4: 31 ;$ y 3:58 horas/día); formación (2:39; 5:51; $2: 55$; y 2:27 horas/día); necesidades básicas (11:16; 11:20; 12:30; y 10:53 horas/día); y tiempo libre (10:07; 9:59; 11:21; y 10:13 horas/día). 
Por otra parte, las pruebas no paramétricas de Welch y Brown-Forsythe sugieren que existen diferencias en la distribución del tiempo profesional (Sig. 0,012 y 0,015 respectivamente), doméstico (Sig. 0,000 para ambas pruebas) y formativo (Sig. 0,000 en ambos casos). Sin embargo, no fue posible demostrar tal circunstancia en relación con las necesidades básicas (Sig. 0,208 y 0,146 respectivamente).

En trabajo profesional las diferencias entre los grupos estadísticamente representativas sólo se producen entre los solteros, que son los que menos tiempo dedican a esta actividad, y los que viven en pareja (Sig. 0,020). Entre el resto de grupos no se puede afirmar que existan comportamientos distintos. Por otra parte, el grupo que más tiempo dedica a las tareas domésticas (los viudos) presenta diferencias significativas con los solteros, que son los que menos tiempo destinan a esta actividad (Sig. 0,000), así como con los individuos que viven en pareja (Sig. 0,001). Estos últimos también presentan diferencias significativas con los solteros (Sig. 0,000). Entre el resto de grupos no puede afirmarse ninguna diferencia con significación estadística. Por último, la formación entre viudos, separados y personas que conviven en pareja no presenta ningún tipo de disimilitud. Sin embargo, el grupo que más tiempo destina a esta actividad, los solteros, presentan diferencias significativas con los tres restantes (Sig. 0,000 en todos los casos).

\subsubsection{Formación}

Esta variable se clasificó en tres grandes grupos: sin estudios o con estudios primarios; formación secundaria (bachillerato, formación profesional o similar); y estudios universitarios, ya sean de grado medio o superior. Los resultados obtenidos se presentan en el Cuadro 4. En éste se aprecia con nitidez que el tiempo dedicado a la actividad profesional aumenta a medida que se posee una titulación académica superior: 1,52, 2,01 y 2,95 horas al día respectivamente. Algo similar, aunque en dirección opuesta, ocurre con el tiempo empleado en las tareas domésticas ( $4,55,3,27$ y 3,06 respectivamente). Paradójicamente, el tiempo dedicado a la formación es insignificante entre las personas que -a priori- más lo necesitan, mientras que aumenta entre los encuestados con estudios de grado medio o superior. Por otra parte, las diferencias entre los grupos con respecto al uso del tiempo destinado a satisfacer necesidades básicas es muy reducida: $8,44,8,39$ y 8,21 horas al día respectivamente. Finalmente, las personas con menor formación parecen disponer de más tiempo libre ( 9,11 horas diarias). La situación cambia en los 
niveles formativos medios (8,60 horas/día) y, sobre todo, entre los titulados universitarios $(8,10 \text { horas/día })^{10}$.

CUADRO 4.

Distribución del tiempo promedio diario según el nivel formativo

\begin{tabular}{|c|c|c|c|c|}
\hline Variables & Nivel de formación & Individuos & Media & $\begin{array}{c}\text { Desviación } \\
\text { típica }\end{array}$ \\
\hline \multirow{4}{*}{$\begin{array}{l}\text { Trabajo } \\
\text { profesional }\end{array}$} & Sin estudios o primarios & 705 & 1,52 & 2,726 \\
\hline & Secundarios & 356 & 2,01 & 2,866 \\
\hline & \begin{tabular}{|l|} 
Superiores \\
\end{tabular} & 239 & 2,95 & 2,741 \\
\hline & Total & 1.300 & 1,92 & 2,816 \\
\hline \multirow{4}{*}{$\begin{array}{l}\text { Trabajo } \\
\text { doméstico }\end{array}$} & Sin estudios o primarios & 705 & 4,55 & 2,795 \\
\hline & Secundarios & 356 & 3,27 & 2,597 \\
\hline & Superiores & 239 & 3,06 & 2,452 \\
\hline & Total & 1.300 & 3,92 & 2,764 \\
\hline \multirow{4}{*}{ Formación } & Sin estudios o primarios & 705 & 0,36 & 1,320 \\
\hline & Secundarios & 356 & 1,70 & 2,696 \\
\hline & \begin{tabular}{|l|} 
Superiores \\
\end{tabular} & 239 & 1,66 & 2,651 \\
\hline & Total & 1.300 & 0,97 & 2,157 \\
\hline \multirow{4}{*}{$\begin{array}{l}\text { Necesidades } \\
\text { básicas }\end{array}$} & Sin estudios o primarios & 705 & 8,44 & 1,427 \\
\hline & Secundarios & 356 & 8,39 & 1,443 \\
\hline & Superiores & 239 & 8,21 & 1,300 \\
\hline & Total & 1.300 & 8,38 & 1,410 \\
\hline \multirow{4}{*}{$\begin{array}{l}\text { Tiempo } \\
\text { libre }\end{array}$} & Sin estudios o primarios & 705 & 9,11 & 2,729 \\
\hline & Secundarios & 356 & 8,60 & 2,678 \\
\hline & \begin{tabular}{|l|} 
Superiores \\
\end{tabular} & 239 & 8,10 & 2,612 \\
\hline & Total & 1.300 & 8,79 & 2,720 \\
\hline
\end{tabular}

Fuente: elaboración propia.

10 El nivel de formación del estudio del INE se agrupa en torno a cinco categorías: a) analfabetos, sin estudios y educación primaria; b) educación secundaria (primera etapa); c) educación secundaria (segunda etapa); d) formación profesional superior; ye) educación universitaria. 
El análisis estadístico sugiere la utilización de pruebas paramétricas en relación con las variables necesidades básicas y tiempo libre. En los demás casos recurrimos a los estadísticos no paramétricos. De este modo, el análisis ANOVA demuestra que las escasas diferencias existentes en relación con el uso del tiempo destinado a satisfacer necesidades básicas no son suficientes para afirmar que el comportamiento entre los tres grupos sea distinto (Sig. 0,094). La conclusión es completamente opuesta por lo que respecta al tiempo libre. En este caso, las diferencias entre los tres grupos son estadísticamente significativas (Sig. 0,000).

El análisis no paramétrico también demostró la existencia de diferencias entre los grupos para las variables restantes: trabajo profesional, trabajo doméstico y formación (los estadísticos de Welch y Brown-Forsythe mostraron en todos los casos significaciones de 0,000 ). En primer lugar, por lo que respecta al trabajo profesional, las diferencias se manifestaron entre los tres niveles formativos (sin estudios o primarios-estudios superiores, Sig. 0,000; estudios secundarios-estudios superiores, Sig. 0,000; sin estudios o primarios-estudios secundarios, Sig. 0,024). Con relación al trabajo doméstico, las diferencias se producen entre el nivel formativo más bajo y los dos restantes (Sig. 0,000), pero no existen conductas diferentes entre los dos grupos con mayor titulación académica (Sig. 0,667). Finalmente, el nivel de estudios de los individuos también condiciona el tiempo dedicado a la formación. Las diferencias se producen, como en el caso anterior, entre el primer grupo y los otros dos, pero no se producen entre éstos (Sig. 0,996).

\subsubsection{Nivel de ingresos}

Otra variable que puede condicionar el modo de distribuir el tiempo es el nivel de ingresos personales. Para realizar este análisis se agrupó dicha información en torno a cuatro niveles de ingresos en términos mensuales: menos de $781,32 €$; de 781,32 a 1.081,82 €; de 1.081,82 a 1.382,33 €; y más de $1.382,33 €$.

En el Cuadro 5 se observa claramente que la dedicación al trabajo profesional aumenta a medida que las personas obtienen unos ingresos superiores. Esta situación se repite con el tiempo destinado a formación. A priori, se podría esperar una tendencia opuesta en relación con el tiempo consumido en las tareas del hogar. Sin embargo, en este caso la dirección no es tan clara como la anterior, ya que se manifiesta con el grupo de mayor nivel económico 
(2,35 horas/día), pero existen algunos solapamientos entre los tres restantes: $3,00,2,81$ y 3,04 horas/día respectivamente. Unas circunstancias similares se producen con el tiempo destinado a satisfacer las necesidades personales y con el tiempo libre ${ }^{11}$.

\section{CUADRO 5.}

Distribución del tiempo promedio diario según ingresos mensuales

\begin{tabular}{|l|l|c|c|c|}
\hline Variables & Ingresos mensuales & Individuos & Media & $\begin{array}{c}\text { Desviación } \\
\text { típica }\end{array}$ \\
\hline \multirow{4}{*}{$\begin{array}{l}\text { Trabajo } \\
\text { profesional }\end{array}$} & Menos de $781,32 €$ & 122 & 4,88 & 2,163 \\
\cline { 2 - 5 } & De 781,32 a $1.081,82 €$ & 128 & 5,26 & 2,362 \\
\cline { 2 - 5 } & De $1.081,82$ a $1.382,33 €$ & 81 & 5,34 & 1,801 \\
\cline { 2 - 5 } & Más de $1.382,33 €$ & 78 & 5,50 & 2,089 \\
\cline { 2 - 5 } & Total & 409 & 5,21 & 2,153 \\
\hline \multirow{4}{*}{$\begin{array}{l}\text { Trabajo } \\
\text { doméstico }\end{array}$} & Menos de $781,32 €$ & 122 & 3,00 & 2,217 \\
\cline { 2 - 5 } & De 781,32 a $1.081,82 €$ & 128 & 2,81 & 2,248 \\
\cline { 2 - 5 } & De $1.081,82$ a $1.382,33 €$ & 81 & 3,04 & 2,254 \\
\cline { 2 - 5 } & Más de $1.382,33 €$ & 78 & 2,35 & 1,995 \\
\cline { 2 - 5 } & Total & 409 & 2,82 & 2,200 \\
\hline \multirow{5}{*}{ Formación } & Menos de $781,32 €$ & 122 & 0,17 & 0,686 \\
\cline { 2 - 5 } & De 781,32 a $1.081,82 €$ & 128 & 0,30 & 0,995 \\
\cline { 2 - 5 } & De $1.081,82$ a $1.382,33 €$ & 81 & 0,46 & 1,161 \\
\cline { 2 - 5 } & Más de $1382,33 €$ & 78 & 0,59 & 1,387 \\
\cline { 2 - 5 } & Total & 409 & 0,35 & 1,049 \\
\hline
\end{tabular}

La distribución del tiempo atendiendo a estos criterios es la siguiente: trabajo profesional (7:46; 7:55; 7:41; 7:52; y 7:18 horas/día, respectivamente); trabajo doméstico (4:06; 3:58; $3: 24 ; 3: 12 ;$ y $3: 01$ horas/día); formación ( $6: 00 ; 5: 50 ; 5: 17 ; 3: 37 ;$ y 4:05 horas/día); necesidades básicas (12:05; 11:05; 10:52; 10:47; y 10:59 horas/día); y tiempo libre (11:02; 10:12; 9:38; 9:24; y $8: 58$ horas/día).

11 En contraste con la presente investigación -que considera el nivel de ingresos personales-, el estudio del INE analiza los ingresos totales del hogar donde convive el entrevistado, estableciendo cuatro rangos: a) menos de $1.000 €$; b) de 1.000 a $1.499,99 €$; c) de 1.500 a $1.999,99$ $€$; y d) $2.000 €$ y más. Los resultados agrupados según estos criterios son los siguientes: 


\begin{tabular}{|l|l|c|c|c|}
\hline Variables & Ingresos mensuales & Individuos & Media & $\begin{array}{c}\text { Desviación } \\
\text { típica }\end{array}$ \\
\hline \multirow{4}{*}{$\begin{array}{l}\text { Necesidades } \\
\text { básicas }\end{array}$} & Menos de $781,32 €$ & 122 & 8,15 & 1,442 \\
\cline { 2 - 5 } & De 781,32 a $1.081,82 €$ & 128 & 7,95 & 1,712 \\
\cline { 2 - 5 } & De $1.081,82$ a $1.382,33 €$ & 81 & 8,13 & 1,300 \\
\cline { 2 - 5 } & Más de $1.382,33 €$ & 78 & 8,03 & 1,520 \\
\cline { 2 - 5 } & Total & 409 & 8,06 & 1,518 \\
\hline \multirow{4}{*}{$\begin{array}{l}\text { Tiempo } \\
\text { libre }\end{array}$} & Menos de $781,32 €$ & 122 & 7,79 & 2,726 \\
\cline { 2 - 5 } & De 781,32 a $1.081,82 €$ & 128 & 7,66 & 2,624 \\
\cline { 2 - 5 } & De $1.081,82$ a $1.382,33 €$ & 81 & 7,00 & 2,579 \\
\cline { 2 - 5 } & Más de $1.382,33 €$ & 78 & 7,51 & 2,248 \\
\cline { 2 - 5 } & Total & 409 & 7,54 & 2,586 \\
\hline
\end{tabular}

Fuente: elaboración propia.

La relación de los ingresos mensuales con las diferentes variables puede analizarse con estadística paramétrica en todos los casos, a excepción de la variable formación (Sig. 0,000). De todos modos, los resultados obtenidos por estos análisis indican que no existen diferencias significativas de ningún tipo, o sea, el nivel de ingresos constituye una variable que no está relacionada con la distribución del tiempo.

\subsection{Situación laboral}

Retomando el objetivo principal de la presente investigación, en este apartado se contrastarán las diferencias existentes en cuanto al empleo del tiempo atendiendo a la situación laboral del individuo. Hay que advertir que la variable trabajo profesional no es considerada en este análisis, ya que el único grupo con posibilidad de dedicar tiempo a ella son los ocupados. Como indicamos anteriormente, el análisis se realiza en tres niveles: a) activos frente a inactivos; b) ocupados versus parados; y c) estudiantes, personas cuya actividad principal son la tareas del hogar ${ }^{12}$ y pensionistas.

trabajo profesional (7:21; 7:44; 7:46; y 7:47 horas/día, respectivamente); trabajo doméstico (4:10; 3:51; 3:38; y 3:13 horas/día); formación (5:18; 5:29; 5:18; y 5:23 horas/día); necesidades básicas (11:54; 11:20; 11:08; y 11:05 horas/día); y tiempo libre (11:13; 10:15; 9:32; y 9:27 horas/día). 


\subsubsection{Activos versus inactivos}

Los activos (ocupados o parados) distribuyen su tiempo tal y como puede apreciarse en el Cuadro $6^{13}$. Es decir, dedican 3,11 horas al día al trabajo doméstico (4,73 los inactivos); 0,58 horas a la formación (1,35 los inactivos); 8,21 horas al día a satisfacer sus necesidades básicas (8,56 los inactivos); y, finalmente, disponen de 8,23 horas por término medio al día para actividades de tiempo libre $(9,34 \text { los inactivos })^{14}$. La apreciación en todos los casos es la misma: los inactivos disponen de más tiempo al día para cada una de las actividades mencionadas. La explicación a este hecho proviene de las restricciones que origina el trabajo profesional entre una parte de la población activa, los ocupados. La distribución del tiempo es una variable de suma cero, de tal manera que lo consumido en ciertas actividades restringe las posibilidades de gasto en otras.

El análisis estadístico de diferencia de medias confirma que el comportamiento de los activos y los inactivos es diferente en relación con la distribución del tiempo en todas las variables analizadas (Sig. 0,000).

\section{CUADRO 6.}

\section{Distribución del tiempo promedio diario: activos versus inactivos}

\begin{tabular}{|l|l|c|c|c|}
\hline Variables & & Individuos & Media & Desviación típica \\
\hline \multirow{2}{*}{$\begin{array}{l}\text { Trabajo } \\
\text { doméstico }\end{array}$} & Activos & 648 & 3,11 & 2,364 \\
\cline { 2 - 5 } & Inactivos & 652 & 4,73 & 2,897 \\
\cline { 2 - 5 } & Total & 1.300 & 3,92 & 2,764 \\
\hline
\end{tabular}

12 En esta situación encontramos abrumadoramente a mujeres. Por este motivo, en los próximos párrafos nos referiremos a ellas como amas de casa.

13 Como hemos indicado, en este caso no tiene sentido la comparación de la variable trabajo profesional, ya que el colectivo de inactivos no desarrolla esta actividad. Esta circunstancia se producirá nuevamente en el próximo apartado, cuando se analicen las diferencias entre los ocupados y los desempleados.

14 Un español activo, según el estudio del INE, dedica 5:04 horas/día al trabajo profesional (inactivos: 0:02), 2:23 horas/día a las tareas domésticas (inactivos: 3:45), dieciséis minutos a formación (los inactivos emplean una hora y veintisiete minutos), consumen 10:48 horas diarias en cubrir sus necesidades básicas (inactivos:12:00) y, por último, disponen de 4:07 horas/día de tiempo libre (inactivos: 5:51). 


\begin{tabular}{|l|l|r|c|c|}
\hline Variables & & Individuos & Media & Desviación típica \\
\hline \multirow{4}{*}{ Formación } & Activos & 648 & 0,58 & 1,554 \\
\cline { 2 - 5 } & Inactivos & 652 & 1,35 & 2,569 \\
\cline { 2 - 5 } & Total & 1.300 & 0,97 & 2,157 \\
\hline \multirow{3}{*}{$\begin{array}{l}\text { Necesidades } \\
\text { básicas }\end{array}$} & Activos & 648 & 8,20 & 1,452 \\
\cline { 2 - 5 } & Inactivos & 652 & 8,56 & 1,345 \\
\cline { 2 - 5 } & Total & 1.300 & 8,38 & 1,410 \\
\hline \multirow{3}{*}{ Tiempo libre } & Activos & 648 & 8,23 & 2,829 \\
\cline { 2 - 5 } & Inactivos & 652 & 9,34 & 2,487 \\
\cline { 2 - 5 } & Total & 1.300 & 8,79 & 2,720 \\
\hline
\end{tabular}

Fuente: elaboración propia.

\subsubsection{Activos: ocupados versus parados}

En el apartado previo se ha demostrado la existencia de comportamientos diferentes en cuanto al empleo del tiempo entre un activo y un inactivo. Pero el análisis debe profundizar en las características de ambos grupos. A continuación evaluaremos la conducta de los activos, ya que es presumible que el modo de distribuir el tiempo de un ocupado sea diferente al de un desempleado.

En el Cuadro 7 se muestra la distribución de ambos colectivos. Los ocupados, al destinar parte de su presupuesto horario a las actividades profesionales, disponen de menos tiempo para el resto de tareas. Las diferencias más apreciables se producen en la dedicación a las labores domésticas (un parado dedica 4,09 horas al día por término medio a esta actividad, frente a las 2,80 de un ocupado) y la disponibilidad de tiempo libre. En este caso, los desempleados "disfrutan" de casi tres horas más al día de tiempo de ocio. En relación con el tiempo destinado a formación y a satisfacer necesidades básicas, las diferencias son similares: un ocupado dispone de alrededor de media hora menos al día para realizar estas tareas ${ }^{15}$.

15 El día promedio de un ocupado, según los resultados del INE, se distribuye del modo siguiente: 5:35 horas al trabajo profesional (desempleados: 0:33); 2:12 horas/día a realizar tareas del hogar (desempleados: 3:58); once minutos al día en formación (desempleados: 59 minutos); 10:43 horas/día en necesidades básicas (desempleados: 11:31); y, por último, disponen de tres horas y cincuenta y tres minutos al día de tiempo de ocio (desempleados: 5:47). 
Además, el análisis de medias confirma las diferencias estadísticamente significativas en todos los casos (Sig. 0,000).

CUADRO 7.

Distribución del tiempo promedio diario: ocupados versus parados

\begin{tabular}{|l|l|c|c|c|}
\hline Variables & Activos & Individuos & Media & Desviación típica \\
\hline \multirow{4}{*}{$\begin{array}{l}\text { Trabajo } \\
\text { doméstico }\end{array}$} & Ocupados & 492 & 2,80 & 2,271 \\
\cline { 2 - 5 } & Parados & 156 & 4,09 & 2,391 \\
\cline { 2 - 5 } Formación & Total & 648 & 3,11 & 2,364 \\
\hline \multirow{3}{*}{$\begin{array}{l}\text { Necesidades } \\
\text { básicas }\end{array}$} & Ocupados & 492 & 0,45 & 1,296 \\
\cline { 2 - 5 } & Parados & 156 & 1,01 & 2,126 \\
\cline { 2 - 5 } & Total & 648 & 0,58 & 1,554 \\
\hline \multirow{4}{*}{ Tiempo libre } & Ocupados & 492 & 8,08 & 1,495 \\
\cline { 2 - 5 } & Parados & 156 & 8,59 & 1,232 \\
\cline { 2 - 5 } & Ootal & 648 & 8,20 & 1,452 \\
\cline { 2 - 5 } & Ocupados & 492 & 7,57 & 2,540 \\
\cline { 2 - 5 } & Total & 156 & 10,29 & 2,703 \\
\hline
\end{tabular}

Fuente: elaboración propia.

\subsubsection{Inactivos: estudiantes, amas de casa y pensionistas}

El colectivo de inactivos, debido a su heterogeneidad (jóvenes estudiantes, amas de casa y personas de edad avanzada, ya jubiladas), también es presumible que presente notables diferencias. En el Cuadro 8 se muestran los resultados obtenidos. En éste se aprecia que las amas de casa son las que dedican más tiempo a las tareas domésticas, como era de esperar (6,49 horas/día). Las diferencias son importantes con los pensionistas ( 3,19 horas/día) y, sobre todo, con los estudiantes, que tan sólo emplean 1,95 horas diarias en estos menesteres. Asimismo, estos últimos están volcados en la formación (5,21 horas/día por término medio), a gran distancia de los pensionistas y las amas de casa. Por su parte, los pensionistas son los que disponen de más tiempo libre, más de once horas al día, y también son los 
que dedican más tiempo a satisfacer sus necesidades básicas (casi nueve horas diarias). En relación con este último aspecto, las diferencias con los estudiantes y amas de casa son pequeñas, exactamente unos treinta minutos más; pero en cuanto al tiempo libre, las distancias se ensanchan, ya que los jubilados disponen de unas tres horas más que el resto de $\operatorname{colectivos}^{16}$.

\section{CUADRO 8. Distribución del tiempo promedio diario de los inactivos}

\begin{tabular}{|l|l|c|c|c|}
\hline Variables & \multicolumn{1}{|c|}{ Inactivos } & Individuos & Media & Desviación típica \\
\hline \multirow{4}{*}{$\begin{array}{l}\text { Trabajo } \\
\text { doméstico }\end{array}$} & Labores hogar & 347 & 6,48 & 1,823 \\
\cline { 2 - 5 } & Estudiantes & 132 & 1,95 & 2,024 \\
\cline { 2 - 5 } & Pensionistas & 130 & 3,18 & 2,422 \\
\cline { 2 - 5 } & Total & 609 & 4,80 & 2,821 \\
\hline \multirow{4}{*}{$\begin{array}{l}\text { Necesidades } \\
\text { básicas }\end{array}$} & Labores hogar & 347 & 0,21 & 0,955 \\
\cline { 2 - 5 } & Estudiantes & 132 & 5,21 & 2,575 \\
\cline { 2 - 5 } & Pensionistas & 130 & 0,34 & 1,229 \\
\cline { 2 - 5 } & Total & 609 & 1,32 & 2,540 \\
\cline { 2 - 5 } & Labores hogar & 347 & 8,49 & 1,306 \\
\cline { 2 - 5 } & Pensionistas & 132 & 8,49 & 1,283 \\
\cline { 2 - 5 } & Total & 609 & 8,93 & 1,318 \\
\hline \multirow{4}{*}{ Tiempo libre } & Labores hogar & 347 & 8,58 & 1,314 \\
\cline { 2 - 5 } & Estudiantes & 132 & 8,34 & 1,942 \\
\cline { 2 - 5 } & Pensionistas & 130 & 11,52 & 2,128 \\
\cline { 2 - 5 } & Total & 609 & 9,28 & 2,427 \\
\hline
\end{tabular}

Fuente: elaboración propia.

16 El INE también distribuye a los inactivos en tres categorías: a) estudiantes; b) jubilados o pensionistas; y c) personas cuya actividad principal son las tareas del hogar. Los resultados obtenidos son los siguientes: trabajo profesional (un minuto; tres minutos; y tres minutos al día, respectivamente); trabajo doméstico (49 minutos; 3 horas y 16 minutos; y 6 horas y 48 minutos); formación (5:26; 0:03; y 0:02 horas/día); necesidades básicas (11:38; 12:41; y 11:24 horas/día); y tiempo libre (4:48; 7:16; y 4:53 horas/día). 
La prueba de homogeneidad de varianzas permite aplicar el análisis paramétrico en relación con la variable necesidades básicas, pero sugiere la utilización de pruebas no paramétricas en los casos restantes. De este modo, el análisis ANOVA confirma la existencia de diferencias estadísticamente significativas entre los diferentes grupos de inactivos en relación con el tiempo empleado en satisfacer las necesidades básicas (Sig. 0,003). Estas diferencias se manifiestan entre el colectivo de jubilados (o sea, los que más tiempo dedican a estos menesteres) y los restantes. Las amas de casa y los estudiantes muestran conductas similares.

Por su parte, las pruebas no paramétricas de Welch y Brown-Forsythe confirman las diferencias entre los distintos colectivos de inactivos para las variables trabajo doméstico, formación y tiempo libre. En relación con el trabajo doméstico, el comportamiento de los tres grupos es distinto en todos los casos (Sig. 0,000). No ocurre lo mismo con las actividades de formación. En esta ocasión, se puede afirmar que la conducta de los estudiantes difiere en comparación con la de los pensionistas y amas de casa (Sig. 0,000 en ambas comparaciones), pero entre estos dos grupos no existen conductas diferentes (Sig. 0,612). Una situación similar se produce en relación con el uso del tiempo libre, aunque en esta ocasión las diferencias se manifiestan entre los jubilados y los colectivos restantes (Sig. 0,000). Por tanto, podemos afirmar que el tiempo de ocio de un estudiante no difiere significativamente del que dispone una ama de casa (Sig. 0,092).

\section{Conclusiones}

El presente trabajo se inició defendiendo que el tiempo es el recurso más valioso del que dispone cualquier persona, así como que el modo de consumir la cantidad disponible varía en función de los valores, gustos y preferencias personales. De todos modos, este consumo está fuertemente condicionado por la situación en la que cada uno se encuentre.

Bajo esta hipótesis, la investigación desarrollada con una muestra de 1.300 personas ha demostrado que existen una serie de variables que condicionan su reparto. Así, utilizando procedimientos estadísticos inferenciales, se han obtenido resultados que sustentan estos argumentos. En la figura siguiente se representan de forma resumida las principales conclusiones obtenidas en relación con las variables que han sido analizadas y la forma de distribuir el tiempo. 


\section{FIGURA 1. Principales conclusiones del estudio}

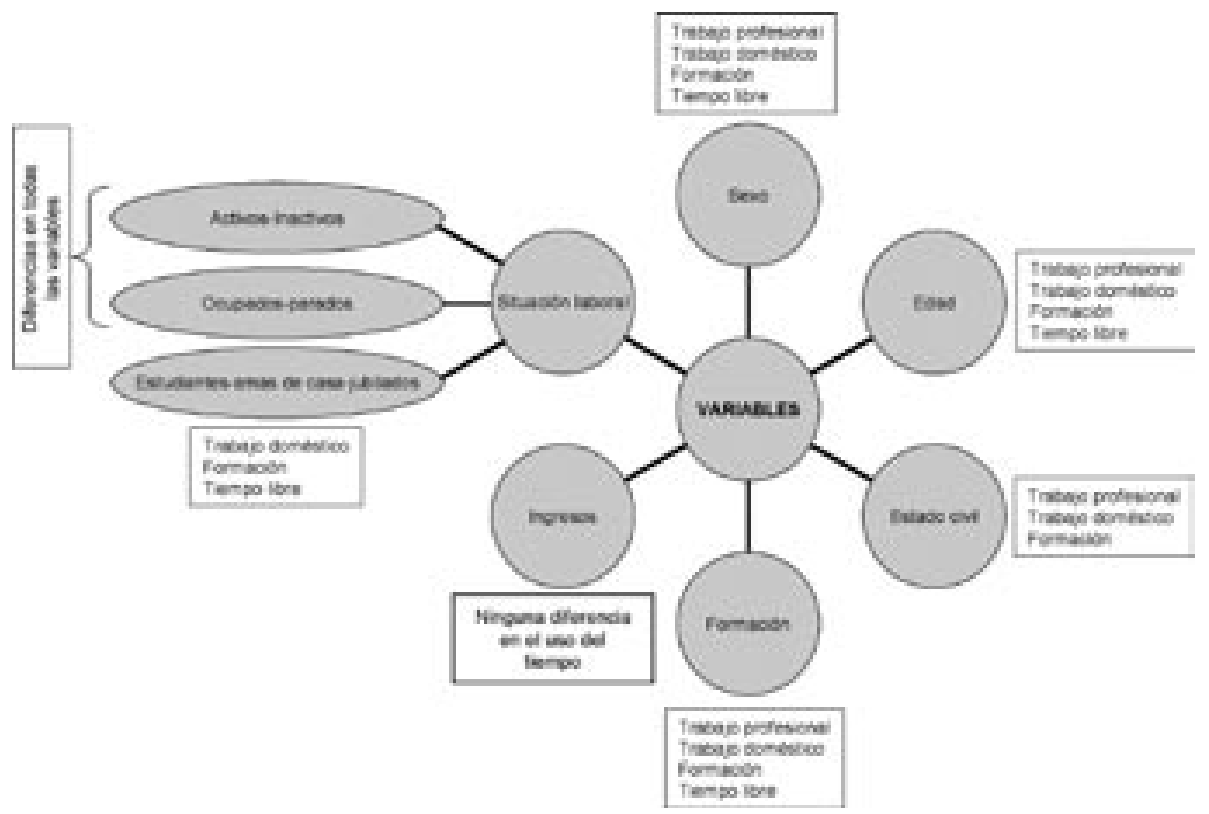

Fuente: elaboración propia.

En primer lugar hay que destacar que todas las variables analizadas se relacionan, en mayor o menor medida, con la forma de distribuir el tiempo, a excepción de la variable "nivel de ingresos", donde no se hallaron diferencias significativas entre los respectivos grupos.

En segundo lugar se aprecia que el tiempo destinado a las necesidades básicas constituye la actividad en la que se producen menos diferencias entre los distintos grupos. Esta circunstancia es lógica, ya que hay una serie de actividades fundamentales -como dormir, comer, asearse, etc.- en las que resulta muy difícil "ahorrar".

Finalmente, en relación con el objeto fundamental de nuestra investigación, se ha constatado que la situación laboral es una variable determinante en la distribución del tiempo diario. Las diferencias entre activos e inactivos, por un lado, y entre ocupados y desempleados, por otro, se manifiestan en todas las actividades diarias: domésticas, tiempo libre, necesidades básicas 
y formación. Entre los diferentes tipos de población inactiva -estudiantes, personas dedicadas a las tareas del hogar y jubilados-las diferencias también se producen en todos los casos, menos en la variable necesidades básicas, por los motivos expuestos anteriormente.

\section{Bibliografía}

AAs, D., (1982), "Designs for Large Scale Time Use Studies of the 24-hour Day", en Z. StaIKov (ed.), It's About Time. Sofia. Institute at the Bulgarian Academy of Sciences, Bulgarian Sociological Association.

Andorka, R. y Falussy, B., (1982), "The Way of Life of the Hungarian Society on the Basis of the Time Budget Survey of 1976-1977", Social Indicators Research, july, 11, 1, pp. 31-73.

Andorka, R., (1987), "Time Budgets and Their Uses". Annual Review of Sociology, $\mathrm{n}^{\circ} 13$, pp. 149-164.

ArIZA, J. A., (2002), El reto del equilibrio: Vida personal y profesional. Una guía práctica para decidir cómo emplear su tiempo. Bilbao, Ed. Desclée De Brouwer. Colección ETEA.

Artiomov, V., (1991), "Cambios en la utilización del tiempo de la población urbana y la rural”. Información Comercial Española, julio, 695, pp. 63-75.

Bittman, M., (1993), “Australian's Changing Use of Time, 1974-1987”, Social Indicators Research, november, 30, 2-3, pp. 91-108.

Clark, S. M. y otros, (1982), "Hypercodes and Composite Variables: Simple Techniques for the Reduction ad Análisis of Time Budget Data", en Z. Staikov (ed.), It's About Time. Sofia. Institute at the Bulgarian Academy of Sciences, Bulgarian Sociological Association.

Eglite, P. y Zarins, I., (1993), "Changes of Time Use of the Town Population in Latvia”, Social Indicators Research, november, 30, 2-3, pp. 109-119.

Gershuny, J. I., (1987), "Vie quotidienne, structure économique et changement technique", Revue Internationale des Sciences Sociales, août, 113, pp. 375-392.

GERSHUNY, J. I., (1991), "International Comparison of time budgets surveys", en Wendy O'CONGHAILE y EBERHARD KÖHLER, The changing use of time. Luxembourg, Office for Official Publications of the European Communities, pp. 12-44. 
GeRSHUny, J. I., (1992), “Are we running out of time?”, Futures, january-february, pp. 3-22.

Gershuny, J. I., (1993), "Post-Industrial Convergence in Time Allocation", Futures, no 25, 5, pp. 578-586.

Gershuny, J. I. y Jones, S., (1986), Time Use in Seven Countries. University of Bath.

HaRvey, A. S. y otros, (1977), Review of Analitic and Descriptive Methods of Time Use Data: A Working Paper. Halifax, Canadá, Institute of Public Affaires. Dalhousie University.

Instituto Nacional de Estadística, (2004), Encuesta de empleo del tiempo 20022003. INE.

Instituto Vasco de Estadística-Eustat, (1998), Demografía y hábitos sociales. Uso social del tiempo. http://www.eustat.es/varios/informes/inf865.pdf

IZQUIERDO, J. y otros, (1988), La desigualdad de las mujeres en el uso del tiempo. Madrid, Instituto de la Mujer.

LundBerg, G., Komarovsky, M. y McInery, M. A., (1934), Leisure: A Suburban Study. New York, Columbia University Press.

PAGE, A., (1996), "Diferencias en el uso del tiempo, entre varones y mujeres y otros grupos sociales". Revista Española de Investigaciones Sociológicas, $\mathrm{n}^{\mathrm{o}} 74$, pp. 291-326.

Pember, M., (1913), Round About a Pound a Week. London, Virago.

Pronovost, G., (1989), “The Sociology of Time”. Current Sociology/Sociologie Contemporaine, winter, 57, 3, pp. 1-129.

RAldúa, E.V., (1997), Presupuestos temporales y cambios en el uso del tiempo. Tesis doctoral. Madrid, Universidad Complutense.

- (2001), "Comparación internacional de los empleos del tiempo de mujeres y hombres”. Revista Española de Investigaciones Sociológicas, nº 94, pp. $105-126$.

Ramos, R., (1990), Cronos dividido. Madrid, CIS (Estudio CIS 1709).

Robinson, J. P., (1985), "Changes in Time Use: an Historical Overview", en F. Thomas Juster y Frank P. Stafford (eds.), Times, Goods and Well-Being. Michigan, Ann Arbor, Univ. Survey Research Center, pp. 289-311. 
Robinson, W. C., (1987), "The Time Cost of Children and Other Household Production", Population Studies. July, 41, 2, pp. 313-323.

Roy, C., (1991), "Évolution des emplois du temps des citadins en France entre 1975 y 1985", en Wendy O'CONGHAILE y EBERHARD KÖLER, The changing use of time. Luxembourg, Office for Official Publications of the European Communities, pp. 186-202.

Samuel, N., (1986), "Évolution de la structure des temps sociaux en France: une transformation des modes de vie", en Société et Loisir, $\mathrm{n}^{\circ}$ 9, 2, pp. 257-278, Québec.

Szalal, A. y otros (eds.) (1972), "The organizational history of the Multinational Comparative Time-Budget Research Project", en AleXander Szalai y otros (eds.), The Use of Time. Paris, Mouton, Daily Activities of Urban and Suburban Populations in Twelve Countries. La Hague, pp. 15-29. 